\title{
Ultrasound as a diagnostic tool in the investigation of patients with carpal tunnel syndrome
}

\author{
Leila Kanafi Vahed (1), Afshin Arianpur (2), Mohammad Gharedaghi (3), Hosein \\ Rezaei (3)
}

(1) Department of Community Medicine, Gilan University of Medical Sciences, Gilan, Iran; (2) Department of Orthopedics, Shohada Hospital, Lahijan, Gilan University of Medical Sciences, Gilan, Iran; (3) Mashhad University of Medical Sciences, Mashhad, Iran

This article is distributed under the terms of the Creative Commons Attribution Noncommercial License (CC BY-NC 4.0) which permits any noncommercial use, distribution, and reproduction in any medium, provided the original author(s) and source are credited.

\begin{abstract}
Carpal tunnel syndrome (CTS) is reveled to be the most common peripheral nerve entrapment syndrome, estimating for $90 \%$ of all compressive. The diagnosis of CTS is based on the use of clinical criteria and imaging technique tests such as ultrasonography (US) and magnetic resonance imaging (MRI). US is a time-saving method in the diagnosis of CTS, which induces less discomfort to the patient and may be a more cost-effective approach to confirm clinical suspicion of this syndrome. The current study was aimed to evaluate the value of US and physical examinations in the diagnosis of CTS. This cross-sectional and cross-sectional prospective case study was conducted to evaluate the usefulness of wrist ultrasonography in diagnosing CTS. Twenty one patients (21 wrists) were invited to participate in the study along with an age- and sex-matched group of participant controls. Physical examination included Phalen, Tinel, Durkan, Tourniquet test. Anteroposterior and mediolateral dimension of carpal tunnel, and the median nerve area at the tunnel were also measured. All the patients underwent the open surgical release of the flexor retinaculum. There was a significant statistical relationship $(p=0.05)$ between anteroposterior diameter of the carpal tunnel and clinical and electro physiologic nerve involvement. Furthermore, some qualitative findings was achieved such as median nerve splitting, hypo echogenicity of the involved nerve, thickening of flexor retinaculum and disappearance of median nerve areas (especially mediolateral direction). In conclusion, ultrasonographic examination of the wrists in the patients with suspected clinical symptoms can improve the diagnostic ability of CTS, especially by improving technology and experience. US can be applied for the median nerve area (MNA) measurement as a first line technique in patients with CTS.
\end{abstract}

Key Words: carpal tunnel syndrome, median nerve, wrist, ultrasonographic examination

Eur J Transl Myol 28 (2): 193-197, 2018

Carpal Tunnel Syndrome(CTS) is one of the most common disorders of the upper limb resulting from the median nerve compression around the wrist at the carpal tunnel arising from repetitive use of the hand. ${ }^{1}$ Many factors contribute to reducing the size of the tunnel or increasing the size of the tissues in the tunnel, leading to CTS symptoms. The prevalence of CTS has been reported to vary from $3.7 \%$ to $5.8 \% .^{2}$ About $10 \%$ of adult women and $1 \%$ of adult males are affected by the disease. The average annual incidence is estimated to be 329 per 100,000 individuals. Patients often experience pain and paresthesis. ${ }^{3,4}$ Risk factors for CTS include advanced age, repetitive use of hand and wrist, obesity, pregnancy, amyloidosis, kidney disease, diabetes mellitus, acromegaly, osteoarthritis, thyroid disease and trauma. ${ }^{5}$ Some studies also found other factors including hormonal factors, the use of Oral Contraceptive Pills (OCP), and its duration, menstrual dysfunction and smoking. ${ }^{6-8}$ Regarding the relatively high prevalence of this syndrome causing significant disability in the hands, timely diagnosis and proper treatment are necessary. The diagnosis of CTS is carried out clinically with a history and physical examination and is confirmed by electrodiagnostic evaluation. High-resolution ultrasonography is used as a useful tool for diagnosis of CTS. The use of US instruments for diagnosis is linked to its broad 


\section{Ultrasound of carpal tunnel syndrome}

Eur J Transl Myol 28 (2): 193-197, 2018

availability, noninvasiveness, shorter enaluation times, and its cost-effective performance. ${ }^{9,10}$ Cross-sectional area (CSA) measurement of medial nerve in the wrist is the most common method of ultrasound for diagnosis of CTS. The normal range for the medial nerve area has been reported at the distal wrist crease from 7.2 to 9.8 $\mathrm{mm}^{2}$, however, the CTS's diagnostic values range from 9 to $15 \mathrm{~mm}^{2}{ }^{11,12}$ A median nerve area (MNA) of $9 \mathrm{~mm}^{2}$ is suggested to have favorable diagnostic value in the distal carpal tunnel by using wrist US. Although many studies have reported a high sensitivity and high profile of wrist ultrasonography, some studies have also expressed dissenting opinions. The most useful clinical trials are those that have a high diagnostic sensitivity and specificity. ${ }^{13,14}$ The sensitivity and specificity of these tests have been different among studies. Many of this variability can be related to various study conditions and measurement methods, along with factors such as gender age and weight. ${ }^{15}$ Evidence have shown that occupational factors play an important role in the disease incidence, especially occupations with more hand-intensive activities. ${ }^{16,17}$ To further explore the usefulness of the MNA US for detecting CTS, the MENA value was measured in patients and control groups if this measurement can predict CTS clinical symptoms in the patient. Therefore, the aim of this study was to evaluate the value of US and physical examinations in the diagnosis of CTS.

\section{Materials and Methods}

\section{Study population}

Two demographic groups were investigated: 1. Case group: Candidates undergoing surgery (CTR) were enrolled in the study, which had classic symptoms of carpal tunnel syndrome during 2001 and 2012; 2. Control group: Women aged 60-60 years were entered into the study without any symptoms of median nerve involvement in upper limb. Two sampling methods were applied: 1. The non-probability sampling (Judgement Sample) for patients; 2. Random sample (Rabdom) for control group. A total of 11 patients (21 wrists) were studied in full, and the data were used in the final analysis. Ten individuals were examined for control group. The study was approved by the Ethics Committee of the Imam Reza Hospital Mashhad. All participants provided a signed informed consent statement.

\section{Data collection}

Patients group. Patients who referred to the clinic with clinical signs of CTS were considered and the following steps were taken. Personal data were recorded in the information form. The Boston Questionnaire was completed by the patient. Then, the self-administered questionnaire was converted into an interview for illiterate patients. The data includes the case number, the date of admission, name, gender, age, occupation, clinical symptoms (surgery in one or both sides, paresthesia, pain, others), symptoms (thenar muscle atrophy, dystrophic changes in the fingers and the underlying causes. The patients were diagnosed clinically through Phalen, Tinel, Durkan, Tourniquet test. Phalen's test was conducted by holding participants hand with wrist in palmar flexion with elbow extended and forearm pronated. Tienel's test has been implemented by applying the median nerve at the wrist. The patient was then sent to conduct electrodiagnostic studies including $\mathrm{NCV}$ test and Eletroneuromyography (EMG) and the results were then included in the information form. Most patients had the results of this experiment in their first exposure. Ultrasound of the wrists was requested and after that, relevant information such as the dimensions of the median nerve inside the carpal tunnel, the dimensions of the carpal tunnel and other possible findings were recorded in the corresponding form. All ultrasound examinations were conducted in similar situations. Candidate patients undergo standard CTR surgery, and evidence was recorded during surgery such as nerve enlargement or Pallid at the tunnel, soft tissue changes. Postoperative patients were discharged with Wellpadded Splint and usually controlled after 10 days. All the patients were satisfied with the clear removal of symptoms, especially the nighttime paresthesia, which confirmed the accuracy of the initial diagnosis. The date of the next referral and the observations were entered in the information form. It is worth noting that patients who did not perform any of the above steps were excluded from the study. 2. Control group. With regard to the agerelated and sexual prevalence of carpal tunnel syndrome (CTS), wrist ultrasounds were performed among women aged 30-60 without any signs of disease. The results of median nerve and carpal tunnel diameters were recorded.

\section{Variables}

1. Ultrasonographic findings of the wrists included median nerve at the carpal tunnel in millimeters (including the anterior posterior and lateral diameter), the dimensions of the carpal tunnel in millimeters (including the anterior posterior and lateral diameters), and dimensions of the carpal tunnel in millimeters (including a smaller anterior posterior diameter and a larger lateral diameter at the Pisiform bone); 2. Different clinical symptoms including paresthesia, pain, night exacerbation, and Phalen, Tinel, and Durkan tests. 3. Electrodiagnostic findings: EMG, NCV tests. 4. Epidemiology: age, sex, one or two sides, occupation, and underlying cause. 5. Surgical findings of median nerve modifications and changes in other soft tissues. 6 . Removing symptoms, especially nighttime paresthesia, in re-referral within the first month after surgery.

\section{Statistical Analysis}

The difference between the meanings was based on zero hypotheses $(\mathrm{H})$. Then, select the appropriate formula ( $\mathrm{t}$ or $\mathrm{Z}$ test). Repeated measures ANOVA model of variance 


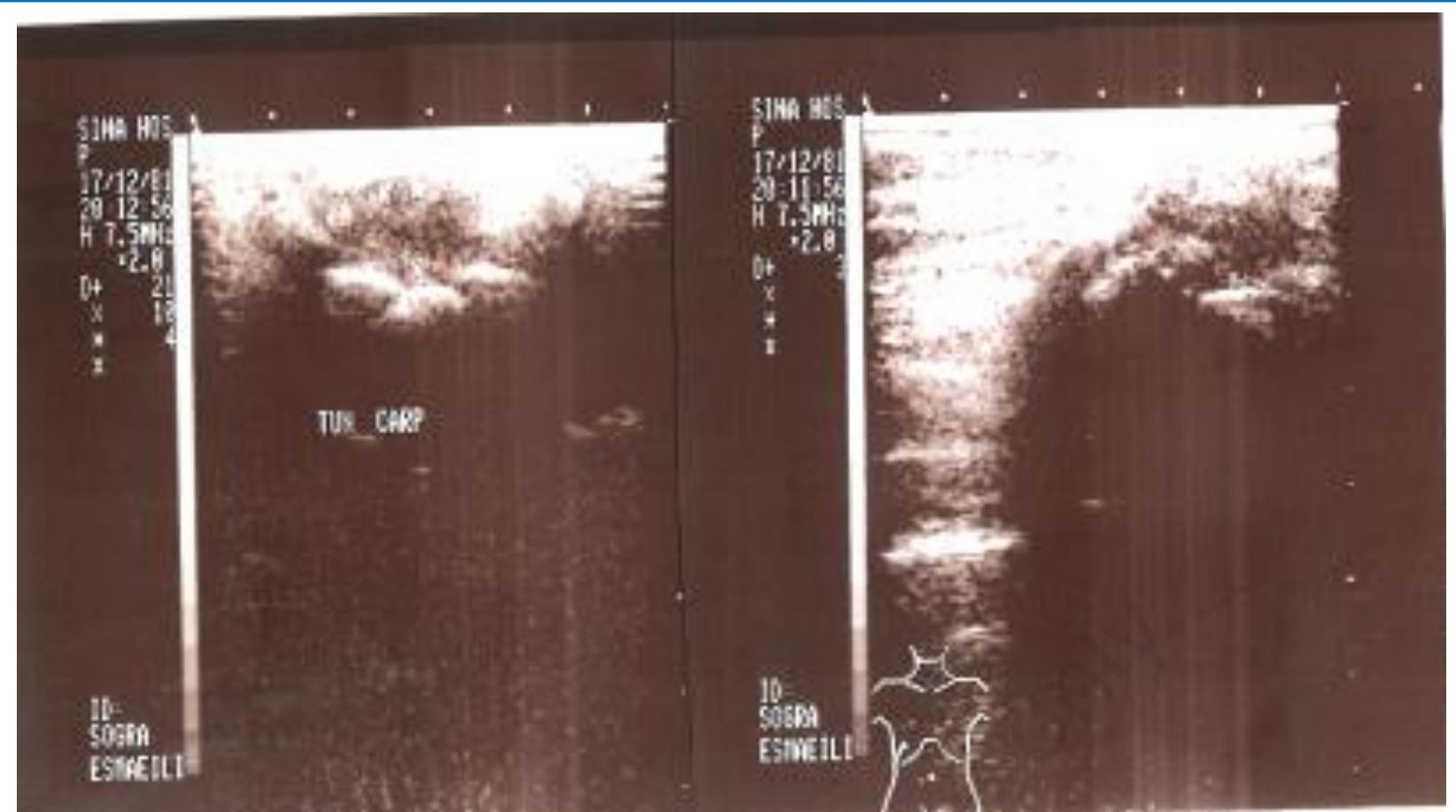

Fig 1. The wrist ultrasound in one of the controls that has been measured in the median nerve and carpal tunnel.

was used to compare reproducibility among different measurements. The significance level was fixed $p<0.5$.

\section{Results and Discussion}

Out of the eleven patients examined, 10 patients were female $(91 \%)$ and one was male. To assess the sexual preference of the patients, statistical tests of $Z$ ratios were performed. The results indicated that there was a significant statistical difference between the two sexes ( $p$ $=0.05$ ). Given the housework of female patients, there were no specific underlying factors, such as repetitive wrist movements, special habits during sleep or history of systemic diseases. The age range of the patients was between the ages of 33 and 62 years (Mean 46, median and mode: 45 years). In all patients, bilateral symptoms also existed, while one-way operation was only performed in one case, since other side had already been acted upon. In terms of the affected side and severity of symptomsat

, 63\% of the cases had symptoms. The severity of the symptoms was predominantly seen in the hands with a prevalence of $63 \%$. Paresthesia was the most common clinical symptom in all patients, where $72 \%$ of cases were associated with nighttime paresthesias. It is not worthy that nighttime paresthesias and insomnia were the main complaint in $73 \%$ of patients. Hand pain was also seen in $81 \%$ of patients. The duration of symptoms is from 2 to 17 years (average 5 years). Interestingly, in almost all cases, despite typical and frequent referral, patients received only oral NSAID treatment or corticosteroid intramuscular injection, while other therapeutic strategies, such as splinting and local injection of cortisone in the early stages of disease, were not applied. They were diagnosed clinically through positive Phalen test that the positive result was obtained in less than 30

Table1. Information of Patients evaluated by ultrasound examination of wrist

\begin{tabular}{llllll}
\hline & Case $(\mathrm{n}=11)$ & & Control(n=11) & & \\
\hline & Diameter(mm) & Mean & Diameter(mm) & Mean & p \\
$\begin{array}{l}\text { Ant.pos median } \\
\text { nerve in carpal } \\
\text { tunnel }\end{array}$ & $3-4$ & 3 & $3-4$ & 3.5 & $=0.05$ \\
$\begin{array}{l}\text { Ant.pos proximity } \\
\text { to pisiform bone }\end{array}$ & $7-10$ & 8 & & & $<0.05$ \\
Lateral & $7-25$ & 21 & $6-11$ & 10 & $>0.05$ \\
\hline
\end{tabular}


seconds in $60 \%$ of cases. Tinel test was positive in $81 \%$ of patients, followed by Durkan test $(90 \%)$ and tourniquet test $(63 \%)$. Different degrees of atrophy and weakness of the thenar muscles were observed in $72 \%$ of patients, while dystrophic finger changes were clinically attributed to a prevalence of $27 \%$. Electrodiagnostic studies included the evaluation of neurotransmission speeds (sensory and motor) and latency calculations. Moreover, the results of electromyography of thenar muscle in all patients showed a degree of median nerve involvement and chronic neuropathy of opponens pollicis muscle. The following information was obtained by wrist ultrasound examination: The anterior posterior diameter of the median nerve at the carpal tunnel was $2.4 \mathrm{~mm}$ (median; $3 \mathrm{~mm}$ ). Due to the fading of the median nerve area due to neuropathic changes, it was not possible to accurately determine the mediolateral diameter of the nerve. The anterior posterior diameter of the carpal tunnel in the pisiform bone was between 7-10 mm (mean, median and mode: $8 \mathrm{~mm}$ ). The lateral diameter of the carpal tunnel was also measured between 17-25 mm (mean $21 \mathrm{~mm}$, median 21, and mode $23 \mathrm{~mm}$ ). In addition to the aforementioned quantitative criteria, symptoms such as fading the median nerve area in most patients, and the reduction of hypoechogenicity, nerve spitting, and thickening of the flexor retinalkolum (each in one case) (Table1). On the other hand, the following information was obtained by examining the ultrasound wrist of the control group: The posterior diameter of the median nerve at the carpal tunnel was defined to be between $3 \mathrm{~mm}$ and $3 \mathrm{~mm}$ (mean and mediation: $3.4 \mathrm{~mm}$ ). Moreover, the median nerve diameter at the carpal tunnel was $3 \mathrm{~mm}$ (mean, median, and mode: $3 \mathrm{~mm}$ ). The anterior posterior diameter of the carpal tunnel adjacent to the pisiform bone was between $6-11 \mathrm{~mm}$ (mean, median and mode: $10 \mathrm{~mm}$ ). The lateral diameter of the carpal tunnel was reported in the same region between 19-21 mm (mean, median, and mode: $20 \mathrm{~mm}$ ). The results of this research have been consistent with the other studies. Recently various studies were performed on the wrist US with measurement of MNA in CTS. In a study by Hobson-Webb et al., ${ }^{18}$ have reported that median nerve US has attached the catalog of potential diagnostic instrumentals and in another studies by Beekman and Visser, 2003 and Wang et al., 2008 have revealed that ultrasound was a crucial tool for the measurement of median nerve in the diagnosis of CTS. ${ }^{15,19}$ Our findings are also in agreement with those of Wiesler et al., 2006, who evaluated forty-four wrists in 26 CTS patients using an ultrasound approach. They demonstrated that highresolution ultrasound is informative in the diagnosis of CTS and indicates enlargement of the median nerve at the distal wrist fold in symptomatic patients as well as they showed that US is good tolerated and safe..$^{20}$ In parallel, Karadağ et al., 2010, have also reported that sonographic measurement in CTS diagnosis could give additional data on extent of involvement of the median nerve. ${ }^{21}$ Do Amaral e Castro et al., 2015, have also documented that
US can be utilized as first option for measuring MNA in CTS. ${ }^{22}$ Our findings of wrists ultrasound between CTS and control group showed that the anterior posterior diameter of the median nerve at the carpal tunnel had no significant difference. However, this result alone is not valuable $(\mathrm{p}=0.05)$. The median nerve diameter at the carpal tunnel was difficult to calculate due to variability in patients caused by neuropathic pressure. Higher visual resolution devices (such as 10 to $20 \mathrm{MHz}$ transducers) and more experiments will possibly accurately measure it. Using our device measurement of the median nerve cross section was not possible. The anterior posterior diameter of the carpal tunnel at levels of pisiform bone between the two groups was statistically significant $(\mathrm{P}=$ 0.05). The lateral diameter of the carpal tunnel between the two groups had not a significant difference. Using qualitative criteria such as fading the median nerve area, increasing the thickening of the flexor retinaculum, decreasing the echogenicity of the affected nerve, and nerve spitting by pressure can somewhat help diagnosis. In conclusion, raw measurements of median nerve dimensions at the carpal tunnel alone are not useful in detecting CTS. It should be measured by calculating the minimum and maximum diameter of the nerve using the proper formula of the nerve cross-section, which involves the use of higher quality instruments and more experience. However, it is reveled that US can be applied for the measurement of MNA as a first line technique in patients with CTS. On the other hand, it was found that the anterior posterior diameter of the carpal tunnel can be used as a diagnostic criterion. Indeed, it was less than 10 $\mathrm{mm}$ (average $8 \mathrm{~mm}$ ) in most patients with a compression neuropathy of the median nerve in the wrists of patients suffering with CTS, while it was usually more than 10 $\mathrm{mm}(\mathrm{p}=0.05)$ in healthy group.

\section{List of acronyms}

CTS - Carpal tunnel syndrome

US - Ultrasonography

MRI - Magnetic resonance imaging

CTR - Candidates undergoing surgery

MNA - Median nerve area

EMG -Eletroneuromyography

\section{Author's contributions}

Authors contributed equally to the manuscript.

\section{Acknowledgments}

None.

Funding: None.

\section{Conflict of Interest}

The authors declare no conflicts of interests.

\section{Ethical Publication Statement}

We confirm that we have read the Journal's position on issues involved in ethical publication and affirm that this report is consistent with those guidelines. 


\section{Ultrasound of carpal tunnel syndrome}

Eur J Transl Myol 28 (2): 193-197, 2018

\section{Corresponding Author}

Afshin Arianpur, Department of Orthopedics, Shohada Hospital, Lahijan, Gilan University of Medical Sciences, Gilan, Iran. Phone: 00989111434497

Email: arian.ortho@yahoo.com

\section{E-mails of co-authors}

Leila Kanafi Vahed: drkanafivahed@yahoo.com Mohammad Gharedaghi: m_gharedaghi@yahoo.com Hosein Rezaei: hoseinrezaei@gmail.com

\section{References}

1. Sung-Bom P, Woohyun S, Seung-Don Y. Slowed conduction velocity of the median sensory nerve across the carpal tunnel in normal adults. Am J Phys Med Rehabil 2005;84:598-603.

2. Atroshi I, Gummesson C, Johnsson R, et al. Prevalence for clinically proved carpal tunnel syndrome is 4 percent. Lakartidningen. 2000; 97:1668-70.

3. Mondelli M, Giannini F, Giacchi M. Carpal tunnel syndrome incidence in a general population. Neurology 2002;58:289-94.

4. Wright PE II. Carpal tunnel, ulnar tunnel, and stenosing tenosynovitis. In: Canale S, Beaty J, editors. Campbell's Operative Orthopaedics. Philadelphia, PA: Mosby Elsevier; 2008. p. $4285-$ 98.

5. Stevens JC, Beard CM, O'Fallon WM, Kurland LT. Conditions associated with carpal tunnel syndrome. Mayo Clin Proc 1992;67:541-48.

6. Bahrmann A, Zieschang T, Neumann $T$, et al. Carpal tunnel syndrome in diabetes mellitus. Med Klin (Munich) 2010;105:150-4.

7. Dias JJ, Burke FD, Wildin CJ, et al. Carpal tunnel syndrome and work. J Hand Surg Br 2004;29:32933.

8. Ferry S, Hannaford P, Warskyj M, et al. Carpal tunnel syndrome: a nested case-control study of risk factors in women. Am J Epidemiol 2000;151:56674.

9. Fowler JR, Gaughan JP, Ilyas AM. The sensitivity and specificity of ultrasound for the diagnosis of carpal tunnel syndrome: a meta-analysis. Clin Orthop Relat Res 2011;469:1089-94.

10. Chen J, Chen L, Wu L, et al. Value of superb microvascular imaging ultrasonography in the diagnosis of carpal tunnel syndrome: Compared with color Doppler and power Doppler. Medicine (Baltimore). 2017;96(21):e6862.
11. Cartwright MS, Shin HW, Passmore LV, Walker FO. Ultrasonographic reference values for assessing the normal median nerve in adults. $\mathbf{J}$ Neuroimaging 2009:19:47-51.

12. Sugimoto $\mathrm{T}$, Ochi $\mathrm{K}$, Hosomi $\mathrm{N}$, et al. Ultrasonographic reference sizes of the median and ulnar nerves and the cervical nerve roots in healthy Japanese adults. Ultrasound Med Biol 2013:39:1560-70.

13. Parikh R, Mathai A, Parikh S, et al. Understanding and using sensitivity, specificity and predictive values. Indian J Ophthalmol 2008;56:45-50.

14. Wiesman IM, Novak CB, Mackinnon SE, Winograd JM. Sensitivity and specificity of clinical testing for carpal tunnel syndrome. Can J Plast Surg 2003;11:70-2.

15. Beekman R, Visser LH. Sonography in the diagnosis of carpal tunnel syndrome: a critical review of the literature. Muscle Nerve 2003;27:2633.

16. Atroshi I, Gummesson C, Johnsson R, et al. Prevalence of carpal tunnel syndrome in a general population. JAMA 1999;282:153-8.

17. Dale AM, Harris-Adamson C, Rempel D, et al. Prevalence and incidence of carpal tunnel syndrome in US working populations: pooled analysis of six prospective studies. Scand J Work Environ Health 2013;39:495-505.

18. Hobson-Webb LD, Massey JM, Juel VC, Sanders DB. The ultrasonographic wrist-to-forearm median nerve area ratio in carpal tunnel syndrome. Clin Neurophysiol 2008:119:1353-7.

19. Wang LY, Leong CP, Huang YC,et al. Best diagnostic criterion in high-resolution ultrasonography for carpal tunnel syndrome. Chang Gung Med J 2008;31:469-76.

20. Wiesler ER, Chloros GD, Cartwright MS, et al. The use of diagnostic ultrasound in carpal tunnel syndrome. J Hand Surg Am 2006;31:726-32.

21. Karadağ YS, Karadağ O, Ciçekli E, et al. Severity of Carpal tunnel syndrome assessed with high frequency ultrasonography. Rheumatol Int 2010;30:761-5.

22. Do Amaral e Castro A, Skare TL, Sakuma AK, Barros WH. Ultrasonography as a tool in diagnosis of carpal tunnel syndrome. Rev Bras Reumatol 2015;55:330-3.

Submission: 06/03/18

Acceptance: $23 / 03 / 18$ 